\title{
EVALUACIÓN MULTICRITERIO DE LAAPTITUD DEL VIARIO EN ESPACIOS NATURALES MEDIANTE SIG
}

\author{
Jesús Vías Martínez, \\ Carmen Ocaña Ocaña \\ Departamento de Geografía. Universidad de Málaga \\ jmvias@uma.es, ocana@uma.es
}

El senderismo constituye una actividad recreativa/deportiva cada vez más demandada por los turistas bajo cualquiera de las variantes de tipos de turismo, pero sobre todo, en el ámbito del turismo rural. Esta actividad demandante de grandes espacios, tiene repercusiones positivas y negativas en el medio. En los últimos años, el elevado crecimiento del senderismo, en determinadas zonas, ha generado impactos de carácter negativo lo que conlleva a tomar medidas que reduzcan los impactos sobre el territorio y la sociedad dependiente de ese medio. En esta línea de conocimiento se enmarca el trabajo que aquí se presenta, el cual permite analizar el territorio de manera que se maximicen los aspectos positivos del senderismo a toda la comunidad vinculada a ese espacio, aspecto que enlaza directamente con la gestión de los espacios naturales, en general, y de los espacios naturales protegidos, en particular.

En este contexto, la evaluación de la aptitud de las infraestructuras viarias para la práctica del senderismo es una técnica que permite analizar la bondad del modelo propuesto para el desarrollo de esta actividad en el medio natural. En este trabajo se ha diseñado una metodología para evaluar la aptitud de la trama viaria de caminos y sendas para la práctica del senderismo, lo cual permitirá proponer futuros senderos de forma que se reduzcan los problemas asociados a la masificación de ciertos sectores y realizar una ordenación del territorio acorde con las necesidades de la zona de estudio.

\section{METODOLOGÍA}

La aptitud del viario para la práctica del senderismo, se ha obtenido mediante una evaluación multicriterio de la aptitud de los tramos que componen la red viaria de la zona de estudio. Esta unidad de análisis (los tramos) la forman cada uno de los segmentos que unen bifurcaciones de la red viaria. Frente a otra posible entidad geográfica o unidad de análisis de la red viaria se ha elegido ésta porque existen físicamente (son visibles) y son funcionales (conectan posibles recorridos). Los tramos de la red viaria utilizados han sido, básicamente, los aportados por la Junta de Andalucía a escala 1/10.000. De esta red de viales, no se han 
utilizado aquellos pequeños tramos que están inconexos y la mayor parte de los cortafuegos. Además, se han añadido tramos cuyo uso senderista es conocido en la zona. Dicho conocimiento procede de las entrevistas mantenidas con usuarios de la zona de estudio y de referencias publicadas en www.wikiloc.com, accesibles desde Google Earth 6.2.2.

A partir de los tramos que conforman la red viaria se ha evaluado la aptitud de cada uno para la práctica senderista en función de tres criterios: Criterio 1: Andabilidad $\left(\mathrm{C}_{1}\right)$, Criterio 2: Interés natural $\left(\mathrm{C}_{2}\right)$ y Criterio 3: Gestión de espacios $\left(\mathrm{C}_{3}\right)$. Cada uno de estos criterios responden a cuestiones relacionadas con la aptitud natural de los caminos para la práctica del senderismo $\left(\mathrm{C}_{1}\right)$, el interés o atractivo que tienen como vía de exploración del territorio $\left(\mathrm{C}_{2}\right)$ y su relación con la planificación que se realiza de los espacios públicos $\left(\mathrm{C}_{3}\right)$, especialmente, los protegidos.

Cada uno de los criterios se evalúa a partir de una serie de factores, los cuales a su vez, son la combinación de diferentes variables. Dado que las variables utilizadas son de naturaleza cualitativa y cuantitativa, se ha procedido en primer lugar a unificar los valores de medida de las variables. En cualquier caso, los valores de todas las variables han sido puntuados entre 0 y 1 , para unificar criterios y mantener la importancia de cada una. Los valores cercanos a 1 indican elevada aptitud para la práctica del senderismo, mientras que los valores cercanos a 0 implican nula aptitud.

Para las variables cualitativas se han utilizado los resultados porcentuales de una encuesta realizada en el marco del proyecto P07HUM-03049, los cuales son fácilmente convertibles a puntuaciones entre 0 y 1 . En el caso de no contar con esta herramienta se han utilizado paneles de expertos. La encuesta se realizó en puntos de inicio de distintas rutas senderistas por la zona de estudio, lo cual a su vez, ha permitido valorar la aptitud de los tramos del viario en función de la opinión de todo el colectivo que hace uso de los senderos estudiados y no solo a partir de la valoración de los «expertos».

Para las variables cuantitativas se ha utilizado el método de normalización de dividir por el máximo. El máximo es el mayor valor teórico que puede alcanzar la variable, independientemente de los valores máximos de la zona de estudio. En caso de que la variable no tenga un máximo, como por ejemplo la longitud de un tramo, se han utilizado intervalos de clase.

En los casos en que el valor de la variable no es homogéneo a todo el recorrido del tramo, el valor que se ha asignado es el valor medio, ponderado por su longitud en la que se obtiene el valor medio.

El valor de aptitud para el criterio $\mathrm{C}_{1}$ (andabilidad) se ha establecido mediante la sumatoria lineal ponderada de las puntuaciones obtenidas por los siguientes cuatro factores: firme, tipo de vía, pendiente media, y obstáculos de pendiente.

- Factor $\mathrm{F}_{11}$ : Firme. Factor relacionado con el tipo de material de que está compuesto el viario teniendo en cuenta el tipo de suelo y el tipo de vegetación que se pisa durante la actividad. Se han diferenciado 4 tipos de firmes: tierra, roca, hierba con roca y hierba con tierra.

- Factor $\mathrm{F}_{12}$ : Tipo de Vías. Factor relacionado con el ancho y el tipo de vía entre los que se han diferenciado caminos y sendas.

- Factor $\mathrm{F}_{13}$ : Pendiente. Este factor indica la pendiente media de los tramos del viario, ordenadas en 5 clases, de llanas a abruptas.

- Factor $\mathrm{F}_{14}$ : Pendiente Obstáculo. Hace referencia a la cantidad de recorrido con una pendiente fuerte $(>30 \%)$ continua que un senderista tiene que superar en un determi- 
nado tramo. Implica un fuerte esfuerzo por parte del senderista, que de mantenerse en una fracción importante del tramo, puede suponer un obstáculo para la realización de la actividad deportiva.

El valor de aptitud respecto al criterio $\mathrm{C}_{2}$ (interés natural), se ha establecido por la sumatoria lineal ponderada de las puntuaciones obtenidas por los factores de entorno vegetal e hitos de interés.

- Factor $\mathrm{F}_{21}$ : Vegetación natural. Este factor tiene en cuenta el paisaje inmediato del sendero, esencialmente las formaciones vegetales del entorno del viario.

- Factor $\mathrm{F}_{22}$ : Hitos de interés. Este factor tiene en cuenta la presencia de hitos de carácter natural o humano, en el entorno del viario, que implican un recurso turístico durante la realización de la actividad senderista.

El criterio $\mathrm{C}_{3}$ relativo a la Gestión, recoge el nivel de protección existente en distintas áreas del parque natural. El criterio se define por un único factor $\left(\mathrm{F}_{31}\right.$ : gestión $)$ y este por una sola variable territorial: la zonificación existente en el parque natural y recogida en el Plan de Ordenación de los Recursos Naturales (PORN) del espacio protegido.

La evaluación de los criterios en un valor de aptitud global se ha realizado mediante la distancia al punto ideal, que refleja la distancia estadística de cada caso respecto a la situación óptima. Esta distancia métrica, por ser una medida de similitud, es más adecuada a este objetivo que la mera agregación lineal, pues la compensación de la media aritmética iguala situaciones de valores de disimilitud diferentes.

El resultado cartográfico de la aptitud se muestra en un mapa mediante tres intervalos con el objetivo de mostrar de forma simplificada la cualidad para el senderismo de los distintos tramos del viario del parque natural. De esta forma se han creado tres intervalos que indican si la aptitud del tramo es Baja, Moderada o Alta para la práctica del senderismo. Los límites de los intervalos se han establecido a partir de la desviación estándar.

\section{RESULTADOS}

La evaluación de la aptitud se ha realizado a los tramos del viario en el Parque Natural Sierra de las Nieves, donde se han analizado 785 tramos de caminos y sendas.

El resultado cartográfico de evaluar la aptitud de los tramos del viario del Parque Natural Sierra de las Nieves manifiesta una elevada aptitud en la zona central del Parque Natural, zona donde se desarrolla en la actualidad la mayoría de las actividades senderistas propuestas en el Plan de Uso Público (PUP) del parque. También se ha obtenido una elevada aptitud en el sector meridional de la zona de estudio, lo que a su vez pone de manifiesto la riqueza de ese sector para la práctica de esta actividad turístico/deportiva, y que no se oferta en el PUP.

Este patrón de localización tiene dos lecturas. Una, la posibilidad de abrir nuevos senderos próximos a los actuales, con la intención de aliviar la carga de los mismos. Otra, la posibilidad de potenciar el uso recreativo de áreas carentes de senderos de uso público, ya prevista pero no materializado en el PUP, especialmente en el sector meridional del parque natural.

En conclusión, la metodología utilizada ofrece la ventaja de estar basada en datos publicados por los organismos públicos, lo cual permite una utilización rápida y económica por parte de los gestores de los espacios naturales. A este respecto, se ha observado que resulta crucial la 
actualización de la cartografía de la red viaria a la escala más detallada que sea posible, con el objetivo de corregir errores derivados de la resolución espacial de los geodatos.

La aptitud de los tramos del viario es una herramienta que permite a los gestores de los Espacios Naturales Protegidos, reordenar la red de senderos del Plan de Uso Público con el fin de descongestionar aquellos sectores con problemas de saturación y dar a conocer nuevas zonas de Parque Natural que aparecen fuera de los circuitos usuales. En el caso de la zona estudiada en este trabajo, la elevada aptitud de los tramos del viario localizados en la parte meridional del Parque Natural que no forman parte de los senderos del Plan de Uso Público, ofrecen una clara oportunidad de puesta en valor para la creación de senderos de elevada aptitud, máxime si se tiene en cuenta la cercanía a una importante fuente de turistas como es la Costa del Sol en las cercanías de Marbella, lo cual abre las posibilidades de un incremento del desarrollo local de los municipios influenciados por el espacio natural protegido. 